\title{
COSMOLOGICAL BACKREACTION
}

\author{
Dominik J. Schwart* \\ Fakultät für Physik \\ Universität Bielefeld \\ Postfach 100131 \\ 33501 Bielefeld, Germany \\ *E-mail:dschwarz@physik.uni-bielefeld.de
}

\begin{abstract}
This work summarises some of the attempts to explain the phenomenon of dark energy as an effective description of complex gravitational physics and the proper interpretation of observations. Cosmological backreaction has been shown to be relevant for observational (precision) cosmology, nevertheless no convincing explanation of dark energy by means of backreaction has been given so far.
\end{abstract}

Keywords: dark energy, structure formation, cosmic expansion

\section{Introduction}

The accelerated expansion of the Universe inferred from the observation of supernovae $(\mathrm{SN}) \mathrm{Ia}^{1]}$ poses one of the biggest puzzles to modern cosmology. While the introduction of a simple term - a cosmological constant — provides a phenomenological explanation, it leads to several conceptual problems at the same time.

Our minimal model, the inflationary $\Lambda$ cold dark matter model, is very successful in describing a large number of cosmological observations like the anisotropies of cosmic microwave background (CMB) radiation, the large scale distribution of galaxies, or the cosmic expansion. Thus it is also called concordance model. Surprisingly, $95 \%$ of the cosmic energy density turn out to be in the form of dark components. Despite the success of the model describing the evolution of the Universe, it obviously fails to explain its content - at least unless we uncover the nature of the dark components.

Especially the nature of the phenomenon of "dark energy" is completely unclear. There are many routes that have to be explored: observational issues, astrophysics issues, testing models of a new component of dark energy, testing modifications to Einstein's equations - to name just a few popular directions. Here I focus on the probably most conservative approach to the problem, assuming that there are no unknown observational and astrophysical issues.

Accelerated expansion of the Universe seems to start when structures of the

${ }^{*}$ Talk given at MG12, Paris 2009 
size of the Hubble scale at matter-radiation equality (the only scale in hierarchical structure formation) have grown mildly non-linear. This suggests that dark energy and structure formation might be linked in some way. It is also well known that the time evolution of cosmic observables and their volume averages do not commute. One might speculate that a mixture of several effects of non-linear structure formation and the fact that we actually observe (light-cone) averages of local quantities could modify the homogeneous and isotropic "background" space-time. One often calls that the idea of cosmological backreaction.

In order to assess the status of these ideas and to motivate their study, I first revisit the key assumptions of the concordance model and stress that some pieces of information come entirely from local data sets $(z<1)$, while some others come from high redshifts at $z>1000$. In section 3 the model-independent evidence for cosmic acceleration is discussed, before we briefly turn to some theoretical issues related to the concordance model and a sketch of various solutions proposed so far. For the rest of this work I focus on the issue of cosmological backreaction, stressing some of the important issues of cosmic structure formation in section 5 and discussing the importance of averaging in section 5. Finally, I summarise some of the open problems.

\section{Concordance model}

The current standard model of cosmology relies four key assumptions:

(1) the standard model of particle physics. It is in excellent agreement with experimental fact - apart from the Higgs, which is searched for at Tevatron and LHC experiments.

(2) general relativity including a cosmological constant. Both geodetic motion and Einstein's field equations are very well tested on various length and time scales. This holds for weak fields (Solar system tests) as well as for strong gravitational fields (pulsars), including the existence of gravitational radiation. The cosmological constant term can only be probed by cosmological observations. It is well motivated by Lovelock's theorem, which tells us that adding a cosmological constant to the Einstein-Hilbert action gives rise to the most general equation of motion for the space-time metric that is covariant and does not contain terms with more than two derivatives. This is a necessary condition for a well posed initial value problem.

(3) the idea of cosmological inflation. It is essential to avoid an enormous amount of fine tuning of initial conditions and to make testable predictions on the geometry of the observable Universe as well as the statistical properties of distribution of matter and light. If inflation lasts for a sufficient number of e-folds, the observable patch of the Universe becomes very close to an isotropic and homogeneous space-time with negligible curvature. Small deviations from isotropy and homogeneity due to quantum fluctuations during inflation are distributed in a statistically isotropic and homogeneous way. As a sufficient amount of e-folds naturally 
occurs for inflationary models with a slow-roll epoch, the power-spectra of matter and metric fluctuations are close to scale invariant. In the simplest models of cosmological inflation, these fluctuations are also gaussian distributed and isentropic (entropy per baryon is constant on spatial hypersurfaces).

(4) the existence of dark matter. There is a plethora of arguments that lead to this conclusion. Some of them are astrophysical, like motion in galaxy clusters or galactic rotation curves. Others are cosmological, like the amount of time necessary for gravitational instabilities to form the observable large scale objects in the Universe. The nature of the dark matter is unknown, candidates are weakly interacting massive particles (beyond the standard model of particle physics), primordial black holes, invisible axions, ... An important property of the dark matter is that it must be cold, which means that its equation of state must be non-relativistic at the onset of structure formation. This property is necessary for sufficiently fast growth of cosmic structures.

The concordance model has 7 free parameters to be tested by our cleanest probe of cosmology: the cosmic microwave background (CMB). These are the photon temperature $T_{0}$, the dimensionless energy density of baryons $\omega_{\mathrm{b}}$ and of cold matter $\omega_{\mathrm{m}}$, the dimensionless Hubble rate $h$, the amplitude of density perturbations $A$, their spectral tilt $n-1$ and the optical depth of microwave photons $\tau$. The optical depth is not a fundamental parameter, and would follow from the other parameters if we had a perfect understanding of astrophysics that leads to the reionisation of the Universe. In the concordance model we have

$$
\Omega_{\Lambda}=1-\frac{\omega_{\mathrm{m}}}{h^{2}}, \quad \Omega_{\mathrm{cdm}}=\frac{\omega_{\mathrm{m}}-\omega_{\mathrm{b}}}{h^{2}}, \quad \Omega_{\mathrm{b}}=\frac{\omega_{\mathrm{b}}}{h^{2}}, \quad \Omega_{\nu}=0 .
$$

Since we know form the detection of neutrino oscillations that neutrinos are massive, one could actually add $\omega_{\nu}$ as an 8th free parameter of the concordance model.

$T_{0}=(2.275 \pm 0.001) \mathrm{K}$, obtained by the COBE FIRAS experiment..$^{2}$ The remaining 6 parameters can be measured from the CMB temperature and polarisation anisotropies alone, the most recent values have been provided by the WMAP team in their analysis of 7 years of data. ${ }^{3}$ This is an impressive consistency check of the model.

The addition of massive neutrinos leads to an upper limit on $\omega_{\nu}<0.014$ (at $95 \%$ C.L.) from the CMB alone,$\sqrt{4}$ but at the same time decreases the best-fit value of $\Omega_{\Lambda}$ by about $10 \%$ and increases the error bars on $\Omega_{\Lambda}, \Omega_{\mathrm{cdm}}$ and $\Omega_{\mathrm{b}}$ by about a factor of two. Still, the concordance model seems to be robust against the addition of neutrino masses.

We may ask if we can also proof some of the key assumptions. The most prominent one (as it is one of the inflationary predictions) probably being the spatial flatness of the Universe. Interestingly enough, this does not follow from the CMB alone. We need to add a local measurement. By local I mean some probe of the Universe at a redshift $z<1$. This has been studied in detail by the WMAP team. It has been shown that either a measurement of $H_{0} \stackrel{6}{6}$ baryon acoustic oscillations 
$(\mathrm{BAOs})^{\sqrt{7}}$ or the Hubble diagram from supernovae (SNe) type $\mathrm{Ia}^{\sqrt[8]{10}}$ can do that. Here I would like to stress, that, in contrast to the primary CMB anisotropies, all of those observables are based on a sample of objects restricted to a volume much smaller than the local Hubble volume.

Another test is to allow for a general equation of state of dark energy ( $w \equiv$ $p / \epsilon \neq-1)$. As in the case of spatial curvature already for a constant $w$ the CMB alone gives only very weak constraints. Combining the $\mathrm{CMB}$ with $H_{0}$, BAO, SN or a measurement of clusters abundances confirms $w=-1$ at the $5 \%$ and $10 \%$ level with and without SNe ${ }^{4}$ Again, we need local $(z<1)$ information to single out the concordance model.

Thus the question arises if modern galaxy redshift surveys, current SN data sets, as well as recent cluster catalogues represent fair samples of the Universe and how much they might differ from the cosmic mean. Let me stress that no survey of large scale structure has ever covered a volume close to the Hubble volume. Typical survey volumes are still well below $V_{\mathrm{H}}$, SN surveys are sometimes pencil beams.

When we add local data sets $(z<1)$ to the CMB, more parameters of astrophysical nature are needed. To give an example, for nearby supernovae their absolute magnitude $M$ must be determined in order to measure the Hubble rate $H_{0}$. Especially for nearby SNe peculiar velocities are important. In order to model them, yet another parameter has to be introduced, which is sometimes included into the error bar on the apparent magnitude.

Adding SNe at larger redshifts $(z \sim 1)$ led to the discovery of cosmic acceleration! 1 This does not require the independent knowledge of $M$ and $H_{0}$. Fitting to models of the cosmic substratum, one only needs a combination of these two parameters, namely $\mathcal{M}=M-5 \log \left(H_{0}\right)+25$ - a quantity that is usually marginalised. Alternatively, one can completely avoid $\mathcal{M}$ by calibrating the $\mathrm{SNe}$ with respect to a low redshift sample of SNe. There is also another hand full of parameters in the light curve fitters, needed to relate the observed SN light curves and spectra to apparent magnitudes in the Hubble diagram. When fitting SNe Ia to the concordance model, one typically constrains one parameter, e.g. $\Omega_{\Lambda}$.

A third increasingly important observable are the baryon acoustic oscillations observed in the local distribution of galaxies. This feature is the late time reflection of the acoustic oscillations seen in the angular power spectrum of the CMB. It is observed at scales of order $100 \mathrm{Mpc}$, thus close to the largest scales that present day surveys can probe. BAOs allows us to probe the angular diameter distance at redshifts $\sim 0.1-1$, but one should keep in mind that the level of sophistication in SN systematics is in advance of BAOs, simply because SNe are being studied since many decades while the BAOs are a very recent subject. As in the case of SNe, some extra parameters have to be added to the concordance model, the most prominent one is the bias $b$, but also others encoding non-linear corrections to the linear power spectrum are important. 


\section{Evidence for accelerated expansion}

The most dramatic consequence of the phenomenon of dark energy, seems to be the accelerated expansion of the Universe. Historically, it has been discovered by ruling out the Einstein-de Sitter model and finding that the $\Lambda$ CDM model provides a much better fit to the SN Ia Hubble diagram. ${ }^{1}$ These observations fell in line with a problematic age estimate on the basis of the Einstein-de Sitter model and thus the majority of researchers quickly adopted the concordance model.

However, ruling out the Einstein-de Sitter model is not the same as finding evidence for acceleration. Already during the "supernova revolution" it was pointed out that an inhomogeneous cosmology could describe the Hubble diagram, 11,13 match the age limits, $\frac{11}{11}$ and - as it turned out later — even match the angular distance scales of the CMB! 14

It is thus very interesting to try to statistically quantify the evidence for acceleration with as few assumptions as possible. The most direct probe of the kinematics of the present Universe are SN Hubble diagrams. In first versions of these tests special parameterizations of the deceleration parameter $q(z) \stackrel{15}{15}$ the scale parameter $a(t)^{16}$ or the Hubble rate $H(z)^{17}$ have been used. Other published methods are to expand $q$ into principle components $\frac{\sqrt{18}}{}$ or to expand the jerk parameter $j$ into a series of orthonormal functions 19

These assumptions are however not necessary, if we would like to test the null hypothesis that the Universe was always decelerating. ${ }^{20121}$ Such a model- and calibration independent test to quantify the evidence for accelerated expansion still needs to rely on the assumptions that the Universe is homogeneous and isotropic and that the SNe of the Hubble diagram are a fair sample of it. The test follows from the simple observation that in a decelerating, flat Friedmann model - independent from its matter content and the validity of Einstein's equations - the luminosity distance satisfies an inequality:

$$
d_{1}(z) \leq(1+z) \ln (1+z) / H_{0} .
$$

This translates to the differential distance modulus $\Delta \mu \equiv \mu_{\mathrm{obs}}-\mu(q=0) \leq 0(q$ is the deceleration parameter), with $\mu \equiv m-M=5 \log d_{1}+25$. The resulting test still depends on a combination of $M$ and $H_{0}$. In order to get rid of this calibration dependence we study $\Delta \mu(z)-\Delta \mu\left(z_{\text {nearby }}\right)$. Some results of this analysis are shown in figure 1

Using the Union compilation of SNe $\mathrm{I}^{8}{ }^{8}$ evidence for cosmic acceleration is found at high significance, which is expressed by the fact that all bins are significantly positive, while they would be expected to be negative for a Universe without acceleration. Summing up all bins, the evidence is $7.2 \sigma \stackrel{21}{21}$ Thus we can conclude that cosmic acceleration is a fact, if we assume isotropy, homogeneity and spatial flatness. The next question is, if we could relax some of these assumptions. Without the assumption of spatial flatness we still found that the evidence for acceleration is $4.2 \sigma$. More critical are the assumptions of homogeneity and isotropy.

What about isotropy? As we know from the $\mathrm{CMB}$, as well as from radio galaxy 


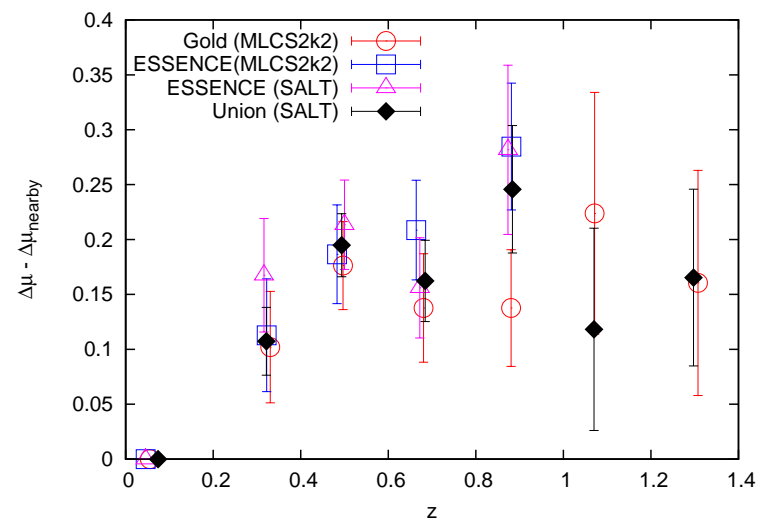

Fig. 1. Cosmic acceleration found at statistically significant levels 21 Strong evidence at $7.2 \sigma$ significance comes from the Union compilation of SNe using the SALT light curve fitter. This analysis assumes that the Universe is isotropic, homogeneous and spatially flat. Dropping the assumption of spatial flatness allows us provides evidence for acceleration at $4.2 \sigma$. The evidence is weaker in case of the MLCS2k2 light curve fitter and for the other data sets shown in the figure. Let me stress the importance of the first data bin (at $z<0.1$ ), which serves as the calibration bin. The error bar of the calibration is taken into account in the error bars shown for the bins at higher redshift.

surveys and other probes of cosmic structure, the deviations from isotropy must be small on large scales. Nevertheless, we can test the SN data. It turns out that they are not as isotropic as one would expect, 22123 but the level of anisotropy found is still consistent with the assumption of isotropy 23 See figure 2 for a hemispherical test of SN Ia Hubble diagrams and their anisotropy. This analysis found an anisotropy in the value of $H_{0}$ at the $10 \%$ level at a statistical significance of $95 \%$ C.L. Note that a $10 \%$ effect in $H_{0}$ could cause the apparent magnitudes to change by 0.2 mag (c.f. figure 1)!

However, the direction of maximal asymmetry coincides with the zenith of the equatorial system, which smells like a systematic issue. Based on other cosmological observations of large scale structure, our bias would be that the Hubble flow should be isotropic, maybe apart from some local bulk flows.

As already discussed above, inhomogeneous cosmologies can fit the SN data, without cosmic acceleration. This does not come as a surprise, as e.g. in the simplest inhomogeneous model - the spherically symmetric Lemaitre-Tolman-Bondi model - we have two free functions at our disposal. Thus the assumption of homogeneity turns out to be absolutely crucial. The homogeneity scale is believed to be at the $100 \mathrm{Mpc}$ scale, ${ }^{24}$ or a redshift of $z>0.1$. We know from observations that huge structures exist, like the Sloan Great Wall, 25 which seems to extend over $\sim 400$ Mpc.

The most vulnerable point in our argument for cosmic acceleration (see figure 11) is thus the calibration bin. We can hardly argue that a sample of SNe at $z<0.1$ can represent a fair sample of the large scale distribution. From that point of view, 


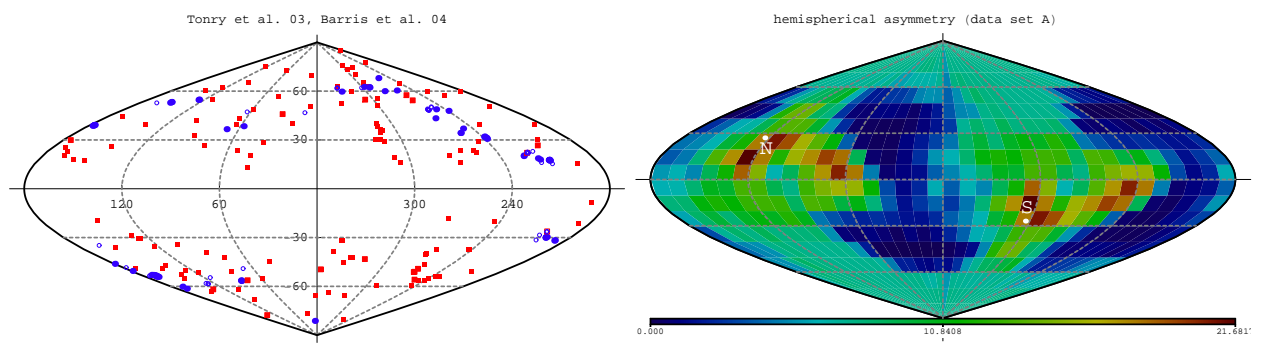

Fig. 2. Statistical (an)isotropy of Hubble diagrams from low redshift SNe ${ }^{23}$ Left panel: Distribution of SNe at $z<0.2$ (red squares) in galactic coordinates. Right panel: Distribution of $\Delta \chi^{2}$ of Hubble diagrams from pairs of hemispheres, centred on the pixel position shown (galactic coordinates). Red regions show hemispherical asymmetries of the Hubble diagram, with $\Delta H_{0} / H_{0} \sim 0.1$, which is statistically significant at the 95\%C.L. The direction of maximal asymmetry is rather close to the zenith of the equatorial coordinate system, and it is thus unclear if this should be regarded as violation of isotropy of local SN data sets or rather as evidence for some systematic issue.

it would be ideal to use $\mathrm{SNe}$ at slightly higher redshifts to calibrate our test. This will be possible with future data set:a

To summarise this section, under the assumption of isotropy and homogeneity of the Universe at all scales, we do find model- and calibration independent evidence for cosmic acceleration. If we allow for prominent local inhomogeneities, one cannot conclude from SN data alone that there is cosmic acceleration.

\section{Dark physics candidates}

Although it is remarkable that a model with 7 free parameters is able to fit the majority of cosmological observations (it does not fit well at the largest angular scales $\left.{ }^{27}\right)$, it has some conceptual problems:

Cosmological constant problem: $: 29$ If we want to understand if some fundamental constant is natural, it is useful to consider dimensionless quantities. There are two fundamental constants of gravity, $\kappa=8 \pi G$ from Newtons constant, and $\Lambda$. Their product is dimensionless and tiny: $\kappa \Lambda \approx 4 \times 10^{-120}$. Already without making any reference to vacuum energy density and quantum field theory, this does not seem to be a natural choice in the context of the gravitational Lagrangian. Moreover, quantum field theory should in principle allow us to calculate the energy density of the vacuum, but it does not work. Attempts to do so end up with estimates of $\kappa \Lambda \sim 1$, which are obviously wrong. Luckily this is no problem in particle physics, as the vacuum energy density would couple to gravity only.

${ }^{\mathrm{a} A}$ preliminary analysis of recent data from SDSS, 10 that would in principle allow that test, shows that the systematic errors from the two different light curve fitters used by the SDSS team lead to conclusions inconsistent with each other? 
Coincidence problem: This is the question of why $\Omega_{\mathrm{de}}\left(t_{0}\right) \sim \Omega_{\mathrm{m}}\left(t_{0}\right)$, where $t_{0}$ is the present age or the Universe. It seems that the present epoch of the Universe is singled out. However, this argument has an anthropic touch as the fact that matter and dark energy are equal at some epoch is not at all a surprising result. There is another formulation of the coincidence problem, which makes only use of scales intrinsic to the concordance models.

Hierarchical structure formation is scale free on small scales (apart form a cut-off at the smallest scales ${ }^{28}$ ), but knows about the matter-radiation equality scale. That is because growth of structures starts at matter-radiation equality and thus there is a change of slope in the density power spectrum and a maximum in the velocity power spectrum at the matter-radiation equality scale. In $\Lambda \mathrm{CDM}$ that scale is at $\sim 100 \mathrm{Mpc}$. Non-linear corrections to the linear perturbation theory start to become important when the density contrast exceeds $\sim 0.3 \mathrm{~b}$. This typically happens at $z_{\mathrm{nl}}[k=1 / 100 \mathrm{Mpc}] \sim z_{\text {acc }} \sim 1$. Thus there is indeed a coincidence, which is independent from mankind.

Many attempts have been made to solve both or one of the mentioned problems. While a solution to the cosmological constant problem probably needs an understanding of quantum gravity, which seems to be out of reach at present, the coincidence problem does not necessarily involve physics that is beyond our scope. The most prominent alternatives to a cosmological constant are:

Dark energy,$\frac{30}{30}$ any component with $p<-\epsilon / 3$ at late times. Specific examples are quintessence, k-essence, Chaplygin gas and many more.

Modified gravity $\frac{31}{3}$ Examples are $f(R)$-models, other curvature invariants, nonminimal couplings, etc.

Effects of cosmic structure $\frac{13[32}{13}$ This includes ideas that violate the Copernican principle by putting us into the centre of the Universe, the idea that the importance of local structures has been underestimated, effects of cosmic averaging and non-linear structure formation itself. Cosmological backreaction belongs to this class of ideas, but sticks to the Copernican principle and to the scenario of inflationary cosmology, as well as to the existence of dark matter.

In the following I will exclusively focus on what I would call cosmic backreaction, some other authors might disagree with my definition of the problem. It seems to me that the most important problem is that we often observe averaged quantities, but make our predictions for the local quantities. This is the so-called averaging problem. An additional complication is that we actually average over a light-cone volume, never over spatial volumes. Not all observables are volume averages, some are averaged over a surface, some just along the line of sight. Now, it is clear that it makes a difference whether we first average a quantity, use that average as the initial condition and then evolve it in time, or if we would start from a complete

${ }^{\mathrm{b}}$ That is when quadratic corrections give rise to $10 \%$ effects. 
knowledge of the initial conditions, evolve them in time and average at the end of that process to compare to observations. It seems to me that after some intermediate irritation in the community there is no doubt left that averaging and evolution do not commute. The dispute is about the order of magnitude of the effect.

\section{Structure formation}

The already mentioned coincidence of the onset of acceleration and the typical scale of large scale structure going non-linear, may provide a hint to ask if dark energy is related to structure formation. 33 [36

In the concordance model we expect that the first structures are seeded by quantum fluctuations during the epoch of inflation. These give rise to acoustic oscillations of the primordial plasma with constant amplitude. Cold dark matter, once kinetically decoupled from the plasma, starts to grow logarithmically on scales smaller than the Hubble horizon during the radiation dominated epoch. Once the Universe becomes matter dominated CDM can grow linearly in the scale factor. This scenario is called hierarchical structure formation as smallest structures form first and they merge and grow to evolve into larger structures. Today scales of $\sim 10$ Mpc have a r.m.s. density contrast of order unity (as $\sigma_{8} \sim 1$ ), while the fluctuations at the horizon scale $(\sim 4000 \mathrm{Mpc})$ are of order $10^{-4}$ in the r.m.s. density contrast. Between these two scales there is the matter-equality scale, which due to the onset of linear growth behaviour is a distinguished scale in structure formation. In the $\Lambda \mathrm{CDM}$ model it is at $\sim 100 \mathrm{Mpc}$. At that scale the density contrast is of order 0.1, thus non-linear effects start to be important. Note that a redshift of 0.1 corresponds to a distance scale of approximately $400 \mathrm{Mpc}$ today, which corresponds to largest observed structures and can thus not be described by linear theory alone.

Observations and simulations based on Newtonian gravity have revealed that the non-linear structure of the Universe forms walls or sheets of matter, which enclose voids, which might be as big as $100 \mathrm{Mpc}$. Within the sheets or at the intersection of sheets filaments are formed, which at their densest spots form clusters and superclusters of galaxies.

An interesting scenario is that our local observations are influenced by this cosmic space-time structure in a way to mimic dark energy. Instead of studying the idea that we are living at the centre of a big over- or underdensity, one can ask what happens if there are many typical pronounced over- or underdensities in the Universe, and we happen to live in one of them. This idea has attracted a lot of attention recently. The problem is that one is limited to toy models of space-time, like the swiss-cheese mode ${ }^{\sqrt{37}}$ or wall-void models ${ }^{\sqrt{38}}$ that are (currently) not derived from first principles.

The upshot of these studies is that some part of the cosmic acceleration seen in SN Hubble diagrams might indeed be due to the effect of a local inhomogeneity although it seems unlikely (see below) that this can explain all of it.

One might argue that dark energy seems to dominate over the dark matter, 
but in the light of the idea of cosmological backreaction this estimate is wrong. In the spirit of cosmological backreaction we can view the cosmic substratum as one component with some effective pressure. The source of acceleration within general relativity is the energy density plus three times the pressure. Todays deceleration parameter is

$$
q_{\mathrm{eff}}=\frac{1}{2}\left(1+3 \frac{p_{\mathrm{eff}}}{\epsilon_{\mathrm{eff}}}\right) .
$$

If compared with the corresponding relation in the $\Lambda$ CDM model,

$$
q_{\Lambda \mathrm{CDM}}=\frac{1}{2}\left(1-3 \Omega_{\Lambda}\right)
$$

we find that $\left|p_{\text {eff }} / \epsilon_{\text {eff }}\right| \sim\left|\Omega_{\Lambda}\right|<1$. Thus, if structure formation should be responsible for the on-set of cosmic acceleration, there is hope to use non-linear perturbation theory to understand this mechanism, at least at higher redshifts and large scales.

\section{Cosmic averages}

Many cosmological observations are averages, e.g. the Hubble constant $H_{0}$. Let us consider an idealised measurement of $H_{0}$ : One picks $N$ standard candles in a local physical volume $V$ (at $z \ll 1$ ), measures their luminosity distances $d_{i}$ and recession velocities $v_{i}=c z_{i}$, and takes the average $H_{0} \equiv \frac{1}{N} \sum_{i=1}^{N} \frac{v_{i}}{d_{i}}$. In the limit of a very big sample, it turns into a volume average $H_{0}=\frac{1}{V} \int \frac{v}{d} \mathrm{~d} V$. For objects at $z \ll 1$, the spatial average is appropriate for the average over the past light cone.

It is still unclear how to best average tensors, $\stackrel{39}{39}$ a way out is to average only scalar quantities. A second problem is to average on the past light cone $\stackrel{40}{4 n}$ the following we restrict our attention to $z \ll 1$, where light-cone averages can be replaced by spatial averages. Calculating spatial averages at the Hubble scale and beyond is not related to any locally observable quantity. It does however make sense to do so at the last scattering surface.

Let me review the formulation of Buchert, $\stackrel{41}{ }$ which has been used to argue that the averaging effect is indeed sizeable and does give important contributions to cosmology. This set up is well adapted to the situation of a real observer. On large scales, a real observer is comoving with matter, uses her own clock, and regards space to be time-orthogonal. These conditions are the definition of the comoving synchronous coordinate system. Buchert used physically comoving boundaries, which is the most natural approach in this setup. In the following the Universe is assumed to be irrotational as a consequence from cosmological inflation.

In synchronous coordinates, the metric of the inhomogeneous and anisotropic Universe is $\mathrm{d} s^{2}=-\mathrm{d} t^{2}+g_{i j}(t, \mathbf{x}) \mathrm{d} x^{i} \mathrm{~d} x^{j}$, and the spatial average of an observable $O(t, \mathbf{x})$ in a physically comoving domain $D$ at time $t$ is defined as

$$
\langle O\rangle_{D} \equiv \frac{1}{V_{D}(t)} \int_{D} O(t, \mathbf{x}) \sqrt{\operatorname{det} g_{i j}} \mathrm{~d} \mathbf{x},
$$


where $V_{D}(t) \equiv \int_{D} \sqrt{\operatorname{det} g_{i j}} \mathrm{~d} \mathbf{x}$ is the volume of the comoving domain $D$. We may introduce an effective scale factor $a_{D}$

$$
\frac{a_{D}}{a_{D_{0}}} \equiv\left(\frac{V_{D}}{V_{D_{0}}}\right)^{1 / 3}
$$

The effective Hubble expansion rate is thus defined as $H_{D} \equiv \dot{a}_{D} / a_{D}=\langle\theta\rangle_{D} / 3(\theta$ being the volume expansion rate).

From the definition (5), we obtain effective Friedmann equations from averaging Einstein's equations,

$$
\left(\frac{\dot{a}_{D}}{a_{D}}\right)^{2}=\frac{8 \pi G}{3} \epsilon_{\mathrm{eff}}, \quad-\frac{\ddot{a}_{D}}{a_{D}}=\frac{4 \pi G}{3}\left(\epsilon_{\mathrm{eff}}+3 p_{\mathrm{eff}}\right),
$$

where $\epsilon_{\mathrm{eff}}$ and $p_{\text {eff }}$ are the energy density and pressure of an effective fluid,

$$
\epsilon_{\mathrm{eff}} \equiv\langle\epsilon\rangle_{D}-\frac{1}{16 \pi G}\left(\langle Q\rangle_{D}+\langle R\rangle_{D}\right), \quad p_{\mathrm{eff}} \equiv-\frac{1}{16 \pi G}\left(\langle Q\rangle_{D}-\frac{1}{3}\langle R\rangle_{D}\right) .
$$

$\langle Q\rangle_{D} \equiv \frac{2}{3}\left(\left\langle\theta^{2}\right\rangle_{D}-\langle\theta\rangle_{D}^{2}\right)-2\left\langle\sigma^{2}\right\rangle_{D}$ is the kinematical backreaction $\left(\sigma^{2}\right.$ being the shear scalar), and $\langle R\rangle_{D}$ the averaged spatial curvature. They are related by the integrability condition

$$
\left(a_{D}^{6}\langle Q\rangle_{D}\right)^{\cdot}+a_{D}^{4}\left(a_{D}^{2}\langle R\rangle_{D}\right)^{\cdot}=0 .
$$

We define the equation of state for the effective fluid as $w_{\text {eff }} \equiv p_{\text {eff }} / \epsilon_{\text {eff }}$. It is highly remarkable that any spatially averaged dust model can be described by an effective Friedman-Lemaître model.

We can map this effective fluid on a model with dust and "dark energy". Let $n$ be the number density of dust particles, and $m$ be their mass. For any comoving domain $\langle n\rangle_{D}=\langle n\rangle_{D_{0}}\left(a_{D_{0}} / a_{D}\right)^{3}$. For a dust Universe, in which $\epsilon(t, \mathbf{x}) \equiv m n(t, \mathbf{x})$, we identify $\epsilon_{\mathrm{m}} \equiv\langle\epsilon\rangle_{D}=m\langle n\rangle_{D}$, and from (8) the dark energy is hence described by $\epsilon_{\mathrm{de}}=-\left(\langle Q\rangle_{D}+\langle R\rangle_{D}\right) /(16 \pi G)$. From (9) we find that constant $\langle Q\rangle_{D}=-\langle R\rangle_{D} / 3$ corresponds to the case of a cosmological constant $\Lambda=\langle Q\rangle_{D}$. Equations (7) and (8) are not closed and additional input is required. Below we close them by means of cosmological perturbation theory.

To study the scale dependence of physical observables $\langle Q\rangle_{D},\langle R\rangle_{D},\langle\epsilon\rangle_{D}, H_{D}$ and $w_{\text {eff }}$, we calculate them to second order in a perturbative series of the effective scale factor $a_{D} \cdot \frac{36 / 42 / 43}{}$ In synchronous gauge, the linear perturbed metric is

$$
\mathrm{d} s^{2}=-\mathrm{d} t^{2}+a^{2}(t)\left[(1-2 \Psi) \delta_{i j}+D_{i j} \chi\right] \mathrm{d} x^{i} \mathrm{~d} x^{j},
$$

where $\Psi$ and $\chi$ are the scalar metric perturbations, $D_{i j} \equiv \partial_{i} \partial_{j}-\frac{1}{3} \delta_{i j} \Delta$ and $\Delta$ denotes the Laplace operator in three-dimensional Euclidean space. The solutions for $\Psi$ and $\chi$ are given in terms of the time independent peculiar gravitational potential $\varphi(\mathbf{x}): \Psi=\frac{1}{2} \Delta \varphi t_{0}^{4 / 3} t^{2 / 3}+\frac{5}{3} \varphi$ and $\chi=-3 \varphi t_{0}^{4 / 3} t^{2 / 3}$ (only growing modes are considered) $\stackrel{36 / 42}{ }$ 
With the help of the integrability condition, we yield the scale dependence of the averaged physical observables up to second order $\underline{43}\left(\langle O\rangle_{D 1} \equiv \int_{D} O \mathrm{~d} \mathbf{x} / \int_{D} \mathrm{~d} \mathbf{x}\right.$ hereafter)

$$
\begin{aligned}
\langle Q\rangle_{D} & =\frac{a_{D_{0}}}{a_{D}} B(\varphi) t_{0}^{2} \\
\langle R\rangle_{D} & =\frac{20}{3} \frac{a_{D_{0}}^{2}}{a_{D}^{2}}\langle\Delta \varphi\rangle_{D 1}-5 \frac{a_{D_{0}}}{a_{D}} B(\varphi) t_{0}^{2} \\
\langle\epsilon\rangle_{D} & =\frac{1}{6 \pi G t_{0}^{2}} \frac{a_{D_{0}}^{3}}{a_{D}^{3}} \\
H_{D} & =\frac{2}{3 t_{0}} \frac{a_{D_{0}}^{3 / 2}}{a_{D}^{3 / 2}}\left[1-\frac{5}{4} \frac{a_{D}}{a_{D_{0}}} t_{0}^{2}\langle\Delta \varphi\rangle_{D 1}+\frac{3}{4} \frac{a_{D}^{2}}{a_{D_{0}}^{2}} t_{0}^{4}\left(B(\varphi)-\frac{25}{24}\langle\Delta \varphi\rangle_{D 1}^{2}\right)\right] \\
w_{\mathrm{eff}} & =\frac{5}{6} \frac{a_{D}}{a_{D_{0}}} t_{0}^{2}\langle\Delta \varphi\rangle_{D 1}-\frac{a_{D}^{2}}{a_{D_{0}}^{2}} t_{0}^{4}\left(B(\varphi)-\frac{25}{12}\langle\Delta \varphi\rangle_{D 1}^{2}\right)
\end{aligned}
$$

with $B(\varphi) \equiv\left\langle\partial^{i}\left(\partial_{i} \varphi \Delta \varphi\right)-\partial^{i}\left(\partial_{j} \varphi \partial^{j} \partial_{i} \varphi\right)\right\rangle_{D 1}-\frac{2}{3}\langle\Delta \varphi\rangle_{D 1}^{2}$. We see from (10) - (14) that these quantities are functions of surface terms only, so all their information is encoded on the boundaries. To a first approximation $a_{D_{0}} / a_{D} \approx 1+z$ and we can see that the perturbative corrections to the kinematic backreaction and to the averaged spatial curvature drop much slower than the matter density. Thus we expect on general grounds of perturbation theory that in a matter dominated Universe, averaged curvature and kinematic backreaction will eventually lead to a breakdown of perturbation theory.

One problem is that it is not simple to observe spatial averages as a function of time. A simpler possibility is to investigate the dependence on the average scale, i.e. the sample volume $V_{D_{0}}^{1 / 3}$. We show below that cosmological averaging produces reliable and important modifications to local physical observables, such as $H_{0}$.

We introduce the quantity 43

$$
\delta_{H}=\frac{H_{D}-H_{0}}{H_{0}},
$$

where $H_{0}$ stands for the true global average, which is well defined in the context of perturbation theory. $H_{D}$ is given by (13). We can now calculate the ensemble average and ensemble variance of $\delta_{H}$. As naively expected the average receives only a contribution form the second order perturbations and is thus small, however the variance receives a contribution from the linear order and is thus sizeable. We find

$$
\sqrt{\operatorname{Var}\left[\delta_{H}\right]} \propto \frac{1}{1+z}\left(\frac{r_{H}}{r}\right)^{2} \sqrt{\mathcal{P}_{\varphi}}
$$

which depends on $H_{0}$ and the dimensionless power spectrum $\mathcal{P}_{\varphi}$, which is fixed by the CMB temperature anisotropies. This is a unique prediction, which can be confronted with data, $\stackrel{43[44}{4}$ as shown in figure 3 ,

It seems to me that one can argue that cosmic averaging is expected to give rise to observable effects. However, this effect alone cannot explain the accelerated ex- 
pansion of the Universe. However, other effects of averaging have not been quantified yet, such as the effects of averaged curvature.

\section{Open problems and no conclusions}

Probably the most important open problem is to learn how to treat light-cone averages in realistic ab initio calculations of the Universe.

My expectation is that the $100 \mathrm{Mpc}$ scale is most important for the effects of cosmic backreaction and that we have to design tests to decide the issue by means of observations. The proposed study of the variance of $\delta_{H}$ is a first idea in that direction. The preliminary conclusion is that cosmic averaging is important, but not necessarily an explanation of dark energy.

However, the hardest challenge for cosmological backreaction is to explain why cosmic backreaction would mimic $\Lambda \mathrm{CDM}$. At the time being, the cosmological constant wins the beauty contest.

\section{Acknowledgements}

I thank Marina Seikel for interesting and fruitful discussions and collaboration and for providing the bottom panel of figure 3 . This work was supported by the Deutsche Forschungsgemeinschaft (DFG).

\section{References}

1. A. Riess A et al., Astron. J. 116 (1998) 1009; S. Perlmutter et al., Astrophys. J. 517 (1999) 565.

2. D. J. Fixsen et al., Astrophys. J. 473 (1996) 576; J. C. Mather et al., Astrophys. J. 512 (1999) 511; D. J. Fixsen and J. C. Mather, Astrophys. J. 581 (2002) 817.

3. D. Larson et al. [WMAP Collaboration], arXiv:1001.4635 [astro-ph.CO].

4. E. Komatsu et al. [WMAP Collaboration], arXiv:1001.4538 [astro-ph.CO].

5. E. Komatsu et al. [WMAP Collaboration], Astrophys. J. Suppl. 180 (2009) 330.

6. A. G. Riess et al., Astrophys. J. 699 (2009) 539.

7. W. J. Percival et al., Mon. Not. R. Astron. Soc. 401 (2010) 2148.

8. M. Kowalski et al. [Supernova Cosmology Project Collaboration], Astrophys. J. 686 (2008) 749.

9. M. Hicken et al., Astrophys. J. 700 (2009) 1097.

10. R. Kessler et al., Astrophys. J. Suppl. 185 (2009) 32.

11. M.P. Dabrowski and M.A. Hendry, Astrophys. J. 498 (1998) 67.

12. J.F. Pascual-Sanchez, Mod. Phys. Lett. A 14 (1999) 1539; K. Tomita, Astrophys. J. 529 (2000) 38; M.-N. Celerier, Astron. Astrophys. 353 (2000) 63;

13. M.-N. Celerier, arXiv:astro-ph/0702416

14. H. Alnes, M. Amarzguioui and O. Gron, Phys. Rev. D 73 (2006) 083519;

15. M. Turner and A. Riess, Astrophys. J. 569 (2002) 18.

16. Y. Wang and M. Tegmark, Phys. Rev. D 71 (2005) 103513.

17. M. John, Astrophys. J. 630 (2005) 667.

18. C. Shapiro and M. Turner, Astrophys. J. 649 (2006) 563.

19. D. Rapetti, S. Allen, M. Amin, and R. Blandford, Mon. Not. R. Astron. Soc. 375 (2007) 1510 . 
20. M. Seikel and D.J. Schwarz, JCAP 0802 (2008) 007.

21. M. Seikel and D.J. Schwarz, JCAP 0902 (2009) 024.

22. M. L. McClure and C. C. Dyer, New Astron. 12 (2007) 533.

23. D.J. Schwarz and B. Weinhorst, Astron. Astrophys. 474 (2007) 717.

24. D.W. Hogg et al., Astrophys. J. 624 (2005) 54.

25. J.R.I. Gott et al., Astrophys. J. 624 (2005) 463.

26. M. Seikel, private communication.

27. D.N. Spergel et al. [WMAP Collaboration], Astrophys. J. Suppl. 148 (2003) 175; A. de Oliveira-Costa, M. Tegmark, M. Zaldarriaga and A. Hamilton, Phys. Rev. D 69 (2004) 063516; H.K. Eriksen et al., Astrophys. J. 605 (2004) 14 [Erratum-ibid. 609 (2004) 1198]; D.J. Schwarz, G.D. Starkman, D. Huterer and C.J. Copi, Phys. Rev. Lett. 93 (2004) 221301; C.J. Copi, D. Huterer, D.J. Schwarz and G.D. Starkman, Mon. Not. Roy. Astron. Soc. 399 (2009) 295; C.L. Bennett et al., arXiv:1001.4758 [astro-ph.CO].

28. S. Hofmann, D.J. Schwarz and H. Stöcker, Phys. Rev. D 64 (2001) 083507; A.M. Green, S. Hofmann and D.J. Schwarz, Mon. Not. R. Astron. Soc. 353 (2004) L23; S. Profumo, K. Sigurdson and M. Kamionkowski, Phys. Rev. Lett. 97 (2006) 031301.

29. S. Weinberg, Rev. Mod. Phys. 61 (1989) 1.

30. P. J. E. Peebles and B. Ratra, Rev. Mod. Phys. 75 (2003) 559; E. J. Copeland, M. Sami and S. Tsujikawa, Int. J. Mod. Phys. D 15 (2006) 1753.

31. R. Durrer and R. Maartens, Gen. Rel. Grav. 40 (2008) 301.

32. T. Buchert, Gen. Rel. Grav. 40 (2008) 467.

33. W. Zimdahl, D.J. Schwarz, A.B. Balakin and D. Pavon, Phys. Rev. D 64 (2001) 063501.

34. D.J. Schwarz, in: "On the Nature of Dark Energy, $18^{\text {th }}$ IAP Astrophysics Colloquium 2002", eds. P. Brax, J. Martin and J.-P. Uzan (Frontier Group, Paris, 2002) p. 331; [astro-ph/0209584]

35. G. Geshnizjani and R.H. Brandenberger, Phys. Rev. D66, 123507 (2002); C. Wetterich, Phys. Rev. D67, 043513 (2003); S. Räsänen, JCAP 0402 (2004) 003; A.A. Coley, N. Pelavas, and R.M. Zalaletdinov, Phys. Rev. Lett. 95, 151102 (2005); E.W. Kolb, S. Matarrese, and A. Riotto, New J. Phys. 8, 322 (2006); T. Buchert, J. Larena and J.M. Alimi, Class. Quant. Grav. 23, 6379 (2006); A. Paranjape and T.P. Singh, Phys. Rev. D76 044006 (2007).

36. E.W. Kolb, S. Matarrese, A. Notari, and A. Riotto, Phys. Rev. D71, 023524 (2005).

37. T. Biswas and A. Notari, JCAP 0806 (2008) 021. V. Marra, E. W. Kolb, S. Matarrese and A. Riotto, Phys. Rev. D 76 (2007) 123004.

38. D.L. Wiltshire, Phys. Rev. Lett. 99 (2007) 251101; B.M. Leith, S.C.C. Ng and D.L. Wiltshire, Astrophys. J. 672 (2008) L91.

39. R.J. van den Hoogen, contribution to this volume; A. Coley, arXiv:1001.0791 [astroph.CO].

40. S. Rasanen, JCAP 0902 (2009) 011; A. A. Coley, arXiv:0905.2442 [gr-qc]; S. Rasanen, arXiv:0912.3370 [astro-ph.CO].

41. T. Buchert, Gen. Rel. Grav. 32 (2000) 105.

42. N. Li and D.J. Schwarz, Phys. Rev. D 76 (2007) 083011.

43. N. Li and D.J. Schwarz, Phys. Rev. D 78 (2008) 083531.

44. M. Seikel and D.J. Schwarz, arXiv:0912.2308 [astro-ph.CO].

45. W. L. Freedman et al. [HST Collaboration], Astrophys. J. 553 (2001) 47.

46. A. G. Riess et al., Astrophys. J. 627 (2005) 579. 

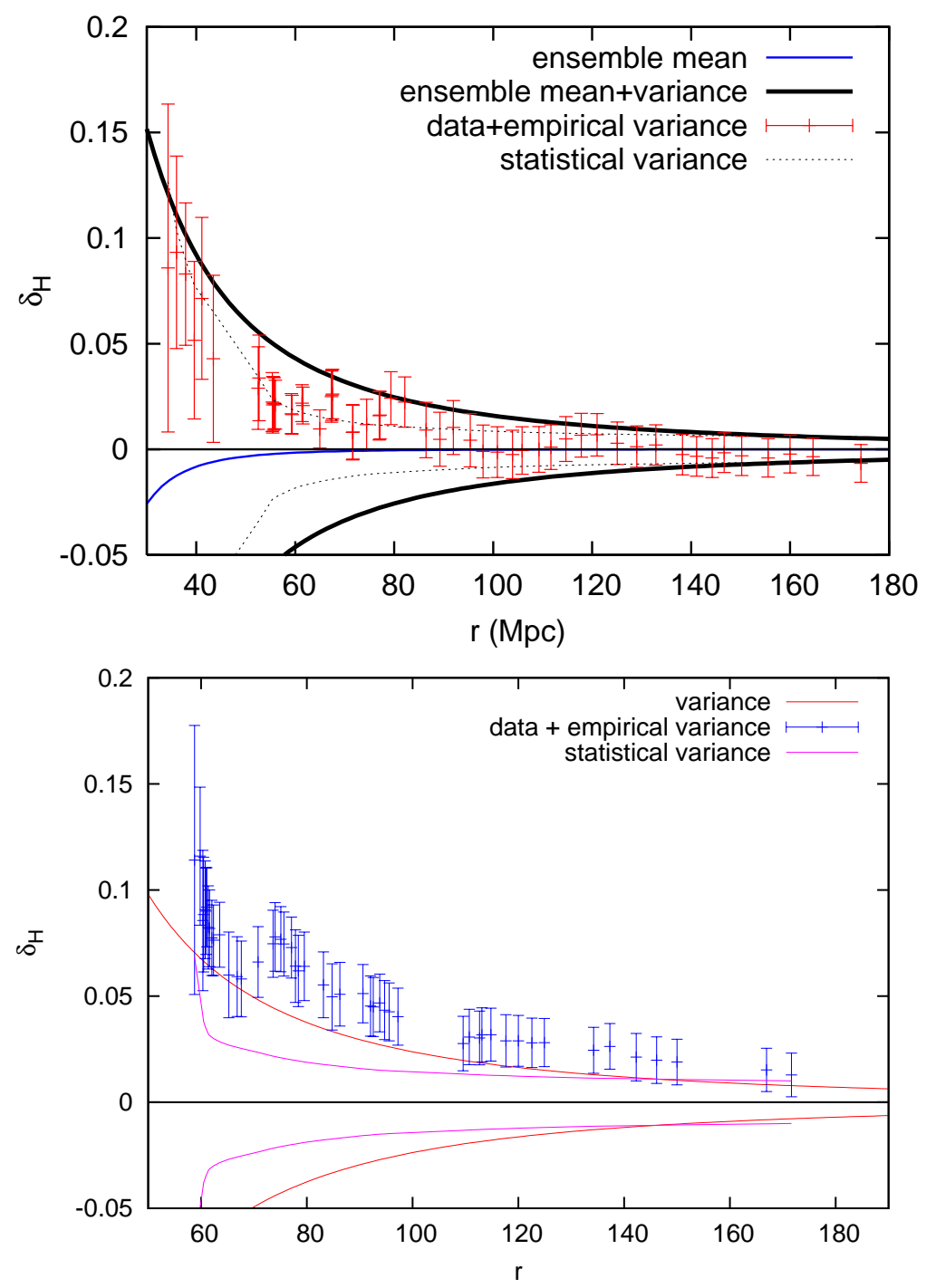

Fig. 3. Volume and sampling dependence of $\delta_{H} \equiv\left(H_{D}-H_{0}\right) / H_{0}$ of observed Hubble diagrams. The ensemble mean of $\delta_{H}$ is negligible at scales $>40 \mathrm{Mpc}$ (blue curve in the top panel). However the ensemble variance of $\delta_{H}$ is $5 \%$ for a spherical volume with $r \sim 60 \mathrm{Mpc}$ and falls with $r^{-2}$ (black line in top panel, red lines in bottom panel). The data averages and their empirical variances are shown from two data sets: data from the Hubble space telescope key project $\frac{45}{4}$ with $H_{0}=72$ $\mathrm{km} / \mathrm{s} / \mathrm{Mpc}$ (top) and the Union compilation of SN $\sqrt{8}$ with $H_{0}=70.5 \mathrm{~km} / \mathrm{s} / \mathrm{Mpc}$ and the calibration suggested in Riess et al!46 (bottom). In both cases the data are fully consistent with the effect of cosmic averaging. While the HST data are also consistent with the hypothesis of pure shot noise, $\delta_{H}(r)$ is well above the expected shot noise between $40 \mathrm{Mpc}$ and $160 \mathrm{Mpc}$ for the local SN form the Union set for the assumed calibration and global value of $H_{0}$. It should be stressed that the latter result suffers from systematic uncertainties regarding the SN calibration. An averaging effect of $5 \%$ in $H_{0}$ could give rise to a shift of the calibrating bin in figure 1 by 0.1 mag. 